\title{
Uma metodologia de localização Indoor para smartphones em ambientes de Cidades Inteligentes.
}

\author{
Hilário José Silveira Castro ${ }^{1}$, Ivanovitch Medeiros Dantas da Silva ${ }^{2}$, Silvio Costa Sampaio ${ }^{2}$ \\ ${ }^{1}$ Universidade Federal do Rio Grande do Norte (UFRN) \\ Programa de Pós-Graduação em Engenharia Elétrica \& Computação \\ Caixa Postal 1524 - 59.078 - 970 - Natal - RN - Brasil \\ ${ }^{2}$ Universidade Federal do Rio Grande do Norte (UFRN) \\ Instituto Metrópole Digital
}

hilariojscastro@gmail.com, ivan@imd.ufrn.br, silviocs@imd.ufrn.br

\begin{abstract}
Indoor localization systems have attracted interests from a wide range of areas, allowing for the optimization and emergence of new services. With the expansion of instrumented scenarios with IoT devices, location systems gain even greater importance, providing references to a location based on the transmitters of the environment. In this work, we present a methodology for an indoor location system for smartphones, capable of tracking the movement of a user and defining their location more precisely, by merging data from different sources and Kalman filters, such as inertial sensors, Wi-Fi and BLE wireless networks installed in the environment.
\end{abstract}

Resumo. Sistemas de localização em ambientes internos (indoor) têm atraído interesses das mais variadas áreas, permitindo a otimização e o surgimento de novos serviços. Com a expansão de cenários instrumentados com dispositivos IoT (do inglês Internet of Things), os sistemas de localização ganham uma importância ainda maior, proporcionando referências para uma localização baseada nos transmissores do ambiente. Neste trabalho, apresentamos uma metodologia para um sistema de localização indoor para smartphones, capaz de rastrear a movimentação de um usuário e definir a sua localização de forma mais precisa, através da fusão de dados de diferentes fontes e Filtro de Kalman, tais como sensores inerciais, redes sem fio Wi-Fi e BLE instalados no ambiente.

\section{Introdução}

A evolução tecnológica dos últimos anos permitiu o surgimento de diversos novos conceitos, entre os quais merecem destaque a Internet das Coisas (do inglês Internet of Things) e as Cidades Inteligentes que, juntos, prometem revolucionar a utilização da tecnologia para o benefício do cidadão. De fato, uma cidade inteligente pressupõe a utilização de uma grande quantidade de dispositivos de IoT, proporcionando a comunicação e interação dos diferentes objetos e atores da cidade, através da Internet [Al-Fuqaha et al. 2015]. Neste cenário, os sistemas de localização indoor (Indoor Location System - ILS) desempenham um importante papel, ao permitir que as informações dos diferentes elementos monitorados possa ser espacialmente referenciada. Por exemplo, ao ativar um serviço inteligente, como algum serviço de emergência, através da aplicação 
de métodos de ILS, é possível determinar a posição de um usuário em uma planta por meio do seu smartphone com uma certa precisão. Este trabalho foi desenvolvido considerando esta situação e objetivando aumentar a precisão das coordenadas geradas. Soluções para este problema são essenciais às Cidades Inteligentes, visto que grande parte dos elementos a serem monitorados certamente estarão em ambientes internos e não em áreas abertas, onde o uso do GPS (do inglês Global Position System) seria uma solução óbvia.

Com uso de sistemas microeletromecânicos nativos de um smartphone, uma ILS pode rastrear a movimentação do usuário e gerar sua posição e coordenadas na planta por meio de métodos como o Pedestrian Dead Reckoning (PDR). Entretanto, imprecisões dos sensores inerciais deturpam as estimações do sistema, acarretando em erros cumulativos no posicionamento do usuário. Uma forma de mitigar deturpações é a hibridização do sistema, fornecendo novas referências para o rastreio [Correa et al. 2017].

A hibridização de uma ILS ocorre com a mescla de sistemas ou técnicas, como adição de redes de transmissores sem fio (wireless) para fornecer novos referenciais ao sistema inercial. Com uso das indicações da potência dos sinais recebidos (Received Signal Strength Indicator - RSSI) de estações sinalizadoras, uma nova coordenada pode ser estimada, servindo de referência extra para o sistema.

O uso de parâmetros de transmissores sem fios, relacionado ao espalhamento de dispositivos sem fios, como referências (âncoras) devem ser tidos em conta já na instalação e disposição destes dispositivos na planta. Dois padrões são popularmente empregados: a família de padrões IEE802.11 (mais conhecida como Wi-Fi) e Bluetooth Low Energy $(B L E)$. Este dois padrões de comunicação são normalmente encontrados em smartphones [Correa et al. 2017].

Neste contexto, este artigo apresenta uma proposta de metodologia de ISL para smartphones. Brevemente, é proposto uma combinação de dados gerados por sistemas inerciais na metodologia PDR com dados derivados da RSSI no sistema de trilateração, formado com base em redes $W i-F i$ e $B L E$, corrigidas por um método de correção de parâmetro. Ao final, os dados serão interpolados por meio de um Filtro de Kalman.

O restante do artigo é dividido em: Seção 2 com trabalhos relacionados; Seção 3 com a descrição da metodologia proposta e detalhes quanto: métodos de rastreio do alvo com uso dos sensores inerciais; propagação de sinais e a distância entre equipamentos; Correção entre distância entre equipamentos e localização por trilateração; e fusão de dados por Filtro de Kalman; Na seção 4 são apresentados alguns testes e análise de resultados; Por fim, a Seção 5 Com a conclusão do artigo e trabalhos futuros.

\section{Trabalhos Relacionados}

Nesta seção, são abordados alguns trabalhos encontrados na literatura relacionados com a construção de sistemas híbridos de localização para smartphones. Em geral, a propostas apresentadas utilizam do multi-sensoriamento do dispositivo, por meio do uso dos sensores para medição inercial, associando a capacidade de realizar comunicação com redes $W i-F i$ ou BLE, entre outros [Correa et al. 2017].

Quanto ao uso de parâmetros de transmissão, uma metodologia comumente utilizada é a fingerprint. Neste método, são registradas as RSSI de todas as estações em cada posição da planta, criando um banco de dados de RSSI. Na resolução, um método de 
associação do banco de dados utiliza as RSSI recebidas posteriormente para determinar a posição. Outras propostas utilizam práticas para aprimorar ou acelerar os resultados, como em [Liu et al. 2012] que utilizou Wi-Fi fingerprints fusionados com dados inerciais por meio de Modelo Oculto de Markov para gerar coordenadas. De forma similar, os autores [Radu and Marina 2013] combinaram dados utilizando de Filtro de Partículas e [Chen et al. 2015] por filtro de Kalman. Outra abordagem está quanto a aproximação entre dispositivos, como nos autores [Chen et al. 2016] que utilizaram de informações de redes $W i-F i$ e $B L E$ para corrigir sistemas inerciais.

Já o uso de fingerprint está associado a precisão em relação a outros métodos baseados em parâmetros de sinais. Entretanto, o método possui como contrapartida um grande esforço na geração e recalibração do mapa de RSSI [Khalajmehrabadi et al. 2017]. Uma formulação com menores necessidades computacionais, menores esforços prévios e sem necessidade de recalibração de mapa é a trilateração. Mesmo com nível de precisão inferior ao fingerprint, a trilateração pode ser utilizada de forma semelhante ao outro método para fusão de dados de redes com sistemas inerciais.

Ao tratar de cenários IoT, um smartphone pode utilizar as estações wireless do ambiente como âncoras ou referências em seus métodos. Duas tecnologias de comunicação nativas no dispositivo e empregadas em dispositivos IoT são o Wi-Fi e $B L E$ [Al-Fuqaha et al. 2015]. Como pode ser observado nas citações, sistemas híbridos trazem uma gama de possibilidades e uma abordagem pouco trabalhada está quanto ao o uso de padrões mistos de transmissão.

Finalmente, neste trabalho é apresentada uma proposta de metodologia para aplicação de uma ILS para smartphones que utiliza uma maior quantidade de dados de diferentes fontes a fim melhorar a precisão das coordenadas geradas, em relação aos métodos descritos listados acima. Na abordagem aqui proposta, as coordenadas serão inicialmente determinadas com base da combinação do método PDR e dados de trilateração. Em adição, será utilizado um sistema misto de âncoras $W i-F i$ e $B L E$, juntamente com um método correção de parâmetro de distância.

\section{Metodologia}

Com base em um cenário IoT instrumentado com transmissores wireless, um usuário, percebido e representado por seu smartphone partirá de um ponto de localização conhecida e terá seu trajeto na planta rastreado pelo sistema. O posicionamento do alvo é definido como uma coordenada em um eixo cartesiano, onde as âncoras Wi-Fi e BLE possuem suas coordenadas conhecidas.

No método, sensores inerciais identificam a movimentação do usuário com uso do sensor acelerômetro, registrando a ocorrência do deslocamento do portador do smartphone. O direcionamento do usuário é rastreado por meio do sensor orientação, que atua como um compasso digital. Ao final, os dados são aplicados ao método PDR, gerando as primeiras coordenadas.

Em paralelo, o sistema coletará as RSSI das estações por 5 segundos, utilizando a média das amostras para cada estação detectada. Com uso de parâmetros associados a cada estação, é estimada a distância entre estações, concluindo as novas coordenadas com uso da Trilateração. Com os dados formulados, o método finaliza por interpolar 
os resultados através do Filtro de Kalman, fornecendo um novo dado relativo entre as formulações.

O fluxograma do método segue como na orientação da Figura 1.

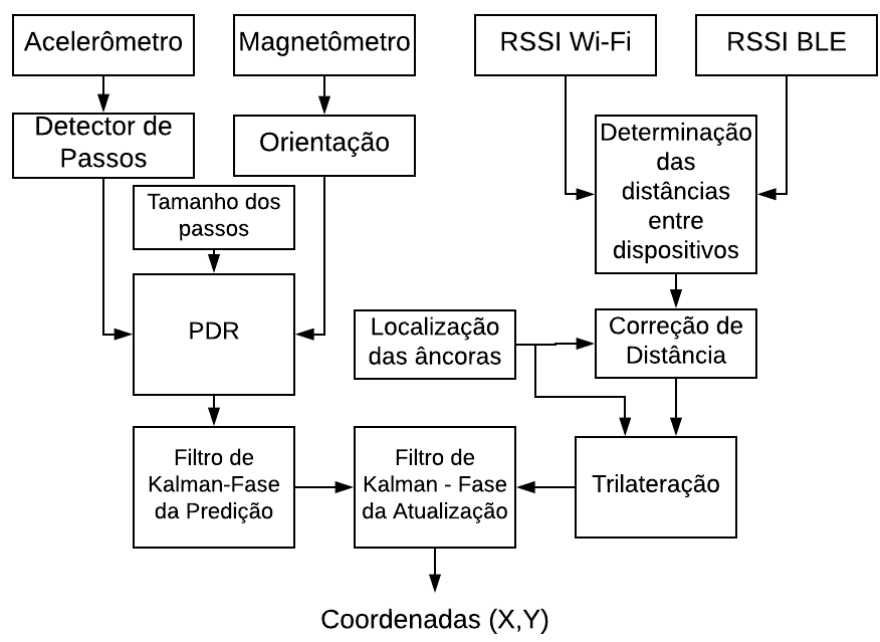

Figure 1. Diagrama de fluxo da metodologia.

\subsection{Sensores inerciais e PDR}

O rastreio da movimentação do usuário é realizado pelo método Pedestrian Dead Reckoning (PDR), descrevendo a posição atual utilizando como base uma posição anterior e o deslocamento realizado [Ojeda and Borenstein 2007]. O método estima as coordenadas atuais $(K)$ de um usuário com base no conhecimento das coordenadas $(X, Y)$ anteriores $(K-1)$, acrescidas do produto da direção tomada em cada eixo $\left(\theta_{K}\right)$ e na quantidade de movimento $\left(d_{K}\right)$ realizado pelo usuário. Como demonstradas pela Equação (1):

$$
\left[\begin{array}{l}
X_{K} \\
Y_{K}
\end{array}\right]=\left[\begin{array}{c}
X_{K-1} \\
Y_{K-1}
\end{array}\right]+\left[\begin{array}{c}
\cos \left(\theta_{K}\right) \\
\operatorname{sen}\left(\theta_{K}\right)
\end{array}\right] d_{K}
$$

A quantidade de movimento e orientação angular são rastreadas por unidades de medição inerciais, em três eixos, contidas no smartphone. Os sensores da unidade requisitados são: Acelerômetro e magnetômetro. Magnetômetro é um sensor utilizado para medição de densidade unidade, indicando a direção e sentido de campos magnéticos em sua proximidade, semelhante a uma bussola, rastreando nos eixos a densidade do fluxo magnético (medido em Tesla ou $A s / \mathrm{m}^{2}$ ) [Milette and Stroud 2012]. Enquanto o acelerômetro atua na para medição da aceleração do dispositivo em $\mathrm{m} / \mathrm{s}^{2}$.

Com o porte do dispositivo na mão do usuário, a detecção de um passo ocorre quando movimentação do usuário implicar na oscilação da aceleração linear em sua mão, durante ato de erguer e repousar o corpo ao dar um passo.

$\mathrm{O}$ ato de erguer o corpo indicará um aumento na aceleração vertical, e o movimento sequencial de repousar indicará uma aceleração em sentido contrário, com os atos rastreados com base nos valores de referências positivos e negativos. Em adição, a identificação de ultrapassagem das referências deve ocorrer em um intervalo de 
tempo semelhante ao tempo de um passo humano, indicado entre $150 \mathrm{~ms}$ e $400 \mathrm{~ms}$ [Jin et al. 2011], a exemplo da contagem de passos da Figura 2. Com a detecção de um passo, a quantidade de deslocamento $\left(d_{k}\right)$ será de $70 \mathrm{~cm}$, que está relativo a um valor médio de um passo humano [Kent 2017].

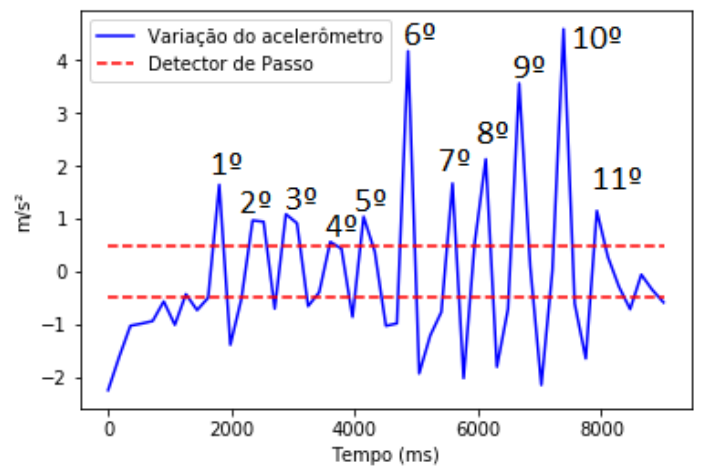

Figure 2. Em uma caminhada de 10 passos o sistema identificou 11 passos.

A mudança da orientação $\left(\theta_{k}\right)$ do usuário será realizada pelo sensor orientação. Este sensor pode monitorar a direção do dispositivo em relação ao norte magnético da terra com uso do acelerômetro e magnetômetro. Entre a formação de 3 eixos no sensor, será utilizado o eixo azimute [Chen et al. 2015]. A leitura do sensor pode ser efetivada por meio de atividades dos eventos dos sensores do dispositivo, como no sistema operacional Android [Milette and Stroud 2012]. Entretanto, os ruídos atrelados aos sensores ocasionam em erros acumulativos para estimação das coordenadas do método PDR, resultando na deturpação da orientação e na quantidade de movimento do usuário, como no exemplo da Figura 2.

\subsection{Estimação da distância entre estações e a Trilateração.}

No dado ambiente, as estações com transmissões wireless enviam sinais a serem captados pelo smartphone. Com uso das RSSI das estações, o dispositivo estimará a distância até o transmissor, por meio da equação (2), baseada no modelo de propagação de sinais Log-distância [Gomes et al. 2013].

$$
d=d_{0} \cdot 10^{\left(\operatorname{Pr}\left(d_{0}\right)-\operatorname{Pr}(d)\right) / 10 . \eta}
$$

Na Equação do modelo tem-se: $\operatorname{Pr}(d)$ como a intensidade de potência do sinal recebido (em $\mathrm{dBm}$ ) a uma dada distância; $P\left(d_{0}\right)$ demonstra a potência do sinal em uma distância de referência $\left(d_{0}\right)$, normalmente a um metro do transmissor; $\eta$ representa a intensidade de desvanecimento do sinal no ambiente, utilizado com intensidade 2 [Rappaport 2001].

Entretanto, os sinais ao propagarem pelo ambiente poderão interagir com barreiras, como estruturas da instalação e o corpo do usuário [Junsheng 2017]. A interação introduz mudanças na intensidade do sinal, seguindo o comportamento de uma variável aleatória gaussiana de média zero com desvio padrão $\sigma$ [Gomes et al. 2013], como exemplificado na Figura 3(a). O efeito descrito, chamado de múltiplos percursos, junto a variação do $\eta$ introduzem os erro na estimação da distância entre estações. 


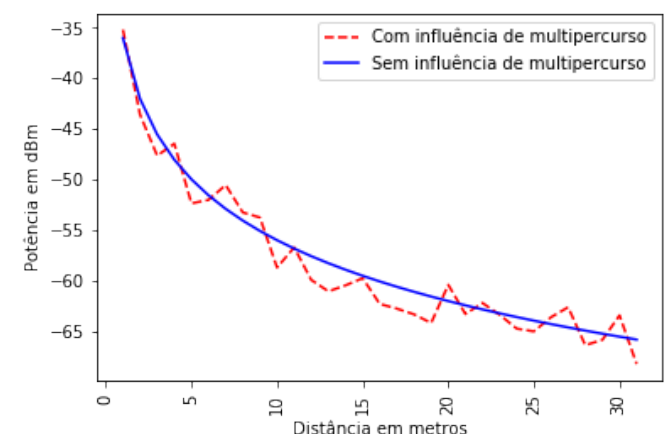

(a) Comparativo entre desvanecimento esperado do sinal e suas mudanças da intensidade da RSSI ocasionadas pelos múltiplos percursos.

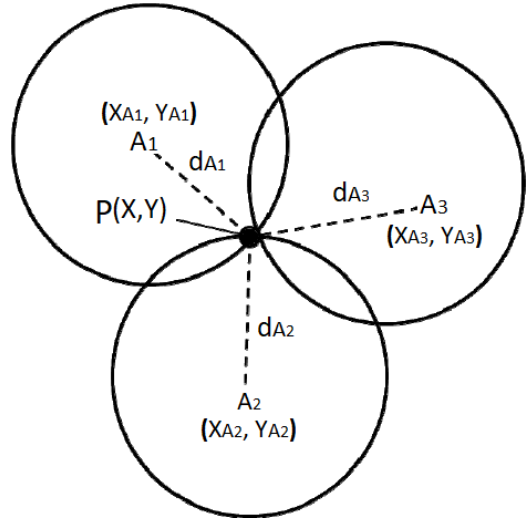

(b) Ponto desconhecido P é o encontro entre três raios formados ao redor de 3 coordenadas conhecidas.

Figure 3.

Utilizando a distância estimada entre dispositivos, aplicasse a Trilateração para determinar as coordenadas do alvo. No principio, é computada a coordenada desconhecida como um ponto de distância comum entre pelo menos três outros pontos (âncoras) de coordenadas conhecidas [Makki et al. 2015], a exemplo da Figura 3(b).

A separação física entre estação receptora e transmissora está relacionada com a distância euclidiana entre os equipamentos. De base no uso necessário de pelo menos 3 pontos, será formado um sistema de Equações (3):

$$
\begin{aligned}
& d_{A_{1}}^{2}=\left(X-X_{A_{1}}\right)^{2}+\left(Y-Y_{A_{1}}\right)^{2} \\
& d_{A_{2}}^{2}=\left(X-X_{A_{2}}\right)^{2}+\left(Y-Y_{A_{2}}\right)^{2} \\
& d_{A_{3}}^{2}=\left(X-X_{A_{3}}\right)^{2}+\left(Y-Y_{A_{3}}\right)^{2}
\end{aligned}
$$

Na Equação (3): $X$ e $Y$ correspondem as coordenadas desconhecidas do ponto móvel; $d_{A_{n}}$ (com $n$ correspondendo aos pontos 1,2 e 3) como a distância entre dispositivo móvel e ponto fixo $n ; X_{A_{n}}$ e $Y_{A_{n}}$ são as coordenadas das estações fixas.

Não obstante, em consequência aos erros na estimação da distância entre estações, as coordenas $(X, Y)$ do alvo terão suas resoluções deturpadas. Para mitigação dos ruídos, será atribuído uma metodologia de correção do parâmetro de distância entre estações, utilizando um sistema de votos. Uma âncora e sua distância para o dispositivo móvel receberá um voto de inadequado seguindo o sistema de Equação (4):

$$
V_{n}=\left\{\begin{array}{rll}
V_{n}, & \text { se } & d_{A_{n} A_{I}}-d_{A_{n}} \leq 1,25 d_{A_{I}} \\
V_{n}+1, & \text { se } & d_{A_{n} A_{I}}-d_{A_{n}}>1,25 d_{A_{I}}
\end{array}\right.
$$

No sistema: $V_{n}$ corresponde a variável de votos de deturpação para uma âncora $n$, com contagem inicialmente nula; $d_{A_{n} A_{I}}$ é a distância euclidiana entre uma âncora avaliada $(n)$ para uma âncora avaliadora $(I) ; d_{A_{n}}$ é a distância entre âncora avaliada e smartphone; $d_{A_{I}}$ distância entre âncora avaliadora e smartphone. 
A âncora com menor número de votos de deturpação $\left(V_{n}\right)$ será considerada a referência do grupo. Com base na âncora de referência, a distância das demais âncoras para o smartphone serão corrigidas seguindo a equação (5), assumindo : $d_{A_{V_{n}}}$ como a distância da âncora de referência para o smartphone e $d_{A_{V_{n}} A_{I}}$ a distância euclidiana de uma âncora $(I)$ para a âncora de referência. Em adição, devem ser eliminadas âncoras com distâncias para o dispositivo móvel maiores que o alcance de suas transmissões no padrão, considerado 10 metros no projeto.

$$
d_{A_{I}}=d_{A_{V_{N}} A_{I}}-d_{A_{V_{N}}}
$$

Com o ajuste nas distâncias finalizado, a resolução do sistema (3) segue utilizando o Método dos Mínimos Quadrados (MMQ) [Anton and Busby 2011].

Com a possibilidade de resultar em diversas âncoras, será aplicada a trilateração em grupos formado por todas as estações fixas, formando $M$ grupos de 3 âncoras, resultando em várias coordenadas $(X, Y)$ diferentes. A coordenada desconhecida será então atrelada a média da soma das coordenadas dos grupos de 3 estações.

\subsection{Filtro de Kalman e a Fusão de técnicas}

Para a interpolação dos dados das metodologias será utilizado o Filtro de Kalman (FK). O FK é um algoritmo de filtro Bayesiano recursivo, capaz de utilizar medições ruidosas ao longo do tempo para gerar resultados que tendem a se aproximar dos valores reais das grandezas medidas [Koo et al. 2014]. O FK é regido por dois grupos de equações: Grupo de Predição e Grupo de Atualização.

Na fase de predição, as Equações utilizam do modelo de dinamismo do sistema associado ao método PDR, como na Equação (6):

$$
X_{K}=X_{K-1}+u_{k}
$$

Na Equação, tem-se: $X_{k}$ como as coordenadas $\left(X_{k}, Y_{k}\right)$ atuais do alvo e $X_{k-1}$ corresponde as coordenadas anteriores; $u_{k}$ descreve a variação das coordenadas $(X, Y)$ entre os estados anterior e atual, fornecida pela transição de coordenadas do PDR. Em sequência, é associado a equação de propagação da covariância do estado ao ruído do modelo, finalizando a etapa de equações de predição, como na Equação (7):

$$
P_{K}^{-}=P_{K-1}+Q
$$

Compõe a Equação (7): $P_{k}^{-}$é Propagação da covariância do estado a priori; $Q$ representa uma variável aleatória com comportamento de ruído gaussiano de média zero no PDR. Seguindo o fluxo das equações, com adição das predições ao o grupo de Atualização. Neste, ocorre a fusão das coordenadas do PDR e da trilateração, expressa em $P_{k}$, como mostram as Equações (8), (9) e (10):

$$
K_{k}=P_{k}^{-}\left(P_{K}^{-}+R\right)^{-1}
$$




$$
\begin{gathered}
X_{k}=X_{k}^{-}+K_{k}\left(Z_{k}-X_{k}^{-}\right) \\
P_{k}=P_{K}^{-}-K_{k} P_{k}^{-}
\end{gathered}
$$

São indicados na Equação: $K_{k}$ como o ganho do filtro de Kalman; $Z_{k}$ indica as coordenadas da trilateração (tr) $\left(X_{t r}, Y_{t r}\right) ; R$ compõe uma variável aleatória com comportamento de o ruído gaussiano de média zero na trilateração; $P_{k}$ fornece as coordenadas fusionadas $(X, Y)$ computadas pela conclusão do filtro. Por meio de $K_{k}$, o FK determinará a contribuição de cada método, adicionando um fator de qualidade as coordenadas de cada método, proporcionados por meio dos ruídos $R$ e $Q$.

Quanto maior a intensidade de $R$ em relação a $Q$, indicará uma confiança maior do método da trilateração em relação ao PDR, o mesmo vale para uma análise inversa, favorecendo o PDR. Outro ponto está relativo ao uso da intensidade dos ruídos diferentes para atrelar resultados melhores em situações diversas. Um exemplo de modificação das intensidades para favorecer os resultados está quanto ao favorecimento para o PDR para casos que existam menos que 3 âncoras para aplicar a trilateração, permitindo o resultado refinado para casos específicos.

\section{Testes e análise de resultados.}

Nesta seção, serão demonstrados alguns testes da proposta. Os dados dos sensores inerciais e das redes foram coletados e armazenados por meio de um aplicativo desenvolvido pelo grupo de pesquisa e aplicada na plataforma Smartphone Samsung Galaxy S4. Os dados armazenados foram aferidos por meio da linguagem de programação Python.

Foram utilizadas como âncoras: 2 estações Wi-Fi Cisco Aironet 1040; e 4 Sensor Tag CC1350 como estações BLE. Os testes ocorreram em uma área aberta com $300 \mathrm{~m}^{2}$, com existência de cadeiras, mesas e passagem de pessoas.

A avaliação do método segue como um comparativo de desempenho entre a proposta e os outros métodos isolados. Nesta avaliação, um usuário portando a plataforma em sua mão segue por um dado caminho. Com os dados coletados pelo dispositivo, cada método irá gerar uma coordenada á cada 5 segundos, formando um trajeto que segue exemplificado nas pelas Figuras 4(a), 4(b) e 4(c).

Em uma análise inicial, é observado o afastamento do método PDR em relação ao trajeto real do usuário ao longo do trajeto estimado. Entretanto, ao associar os dados da Trilateração, mesmo com menor precisão, recondiciona a direção do rastreio do trajeto.

Para uma análise mais complementar, é analisado o desempenho dos métodos pela precisão, comparando a distância euclidiana entre a coordenada real e a coordenada estimada de cada formulação. Na avaliação, foram levantados dados de 20 trajetos idênticos as das Figuras 4(a), 4(b) e 4(c), utilizando como parâmetro avaliativo a posição final do usuário no trajeto. São levantados os erros máximo, mínimo e médio de precisão (em metros) de cada método, registrados na Tabela 1.

Como pode ser observado, o método proposto demonstra uma melhor aproximação das coordenadas geradas em comparação aos outros métodos. O método PDR possui um menor erro a curto prazo, entretanto os erros acumulativos deturpam o posicionamento real do alvo. Embora apresente menor precisão, a trilateração fornece 


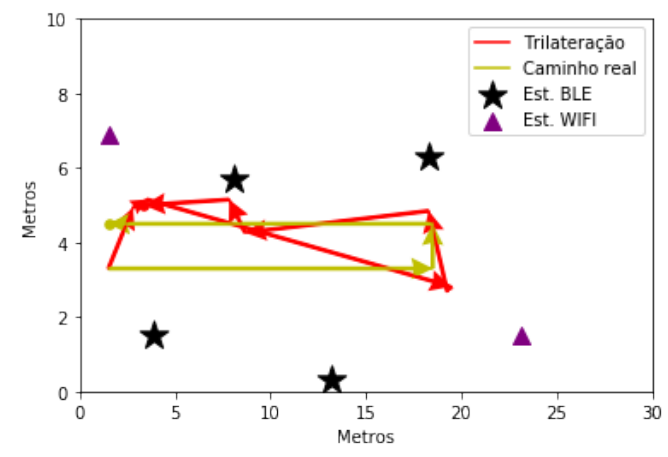

(a) Caminho Real x Trilateração.

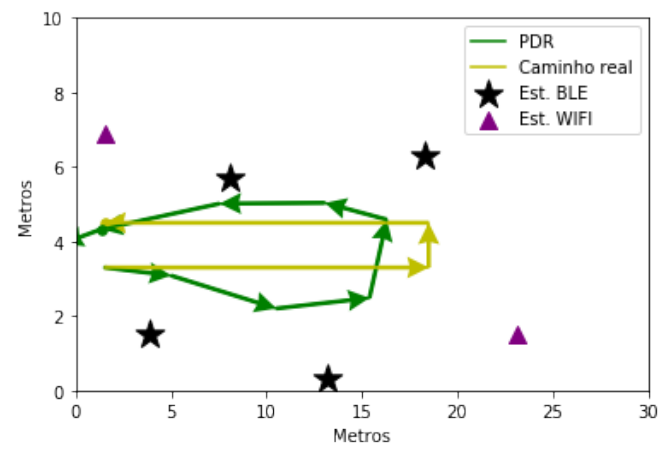

(b) Caminho Real x PDR.

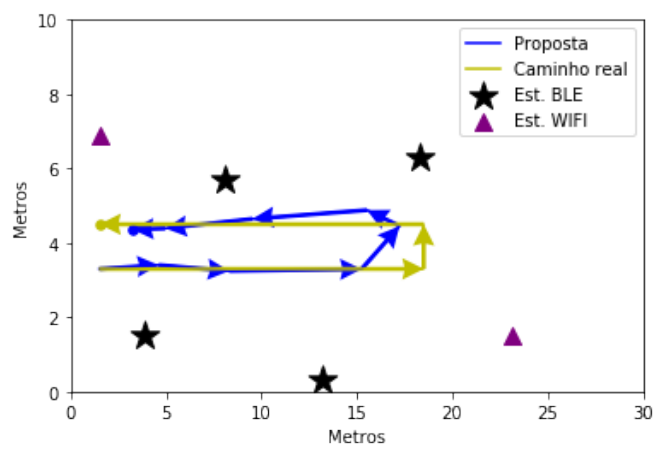

(c) Caminho Real x Método proposto.

Figure 4.

novos referenciais para utilização no método proposto. A combinação dos dados resultam em estimações das coordenadas do alvo mais próximas do caminho real.

Uma vantagem a ser destacada do método em relação a outras metodologias descritas na seção 2, está quanto aos esforços de recalibração, graças a não necessidade de projetar um mapa de RSSI do ambiente, principalmente em grandes granularidades. No método é ocasionado apenas a necessidade de atualização das coordenadas das âncoras e seus parâmetros em situações de mudanças do ambiente.

Table 1. Erros de estimação (em metros) de cada metodologia.

\begin{tabular}{|c|c|c|c|}
\hline Método & Máximo & Minimo & Média \\
\hline Trilateração & $5,872 \mathrm{~m}$ & $1,258 \mathrm{~m}$ & $2,767 \mathrm{~m}$ \\
\hline PDR & $4,216 \mathrm{~m}$ & $0,801 \mathrm{~m}$ & $2,384 \mathrm{~m}$ \\
\hline Proposta & $3,188 \mathrm{~m}$ & $0,417 \mathrm{~m}$ & $1,224 \mathrm{~m}$ \\
\hline
\end{tabular}

\section{Conclusão e Trabalhos Futuros.}

Este trabalho discutiu e apresentou uma proposta de metodologia de localização indoor para smartphones. Resultados preliminares indicaram um bom desempenho da metodologia, a partir da reorientação do método PDR por novas referências. Entretanto, em alguns casos, o método proposto apresentou desempenho inferior em algumas a uma das outras resoluções, indicando uma necessidade de refinamento do projeto. Assim, parte dos futuros esforços do projeto estarão voltados a uma melhoria na sintonia do Filtro de Kalman. 
O aprimoramento do filtro seguirá por meio do controle dos parâmetros $\mathrm{R} \mathrm{e} \mathrm{Q}$, sendo adicionado o uso de outras versões do Filtro de Kalman.

\section{References}

Al-Fuqaha, A., Guizani, M., Mohammadi, M., Aledhari, M., and Ayyash, M. (2015). Internet of things: A survey on enabling technologies, protocols, and applications. IEEE Communications Surveys Tutorials, 17(4):2347-2376.

Anton, H. and Busby, R. (2011). Álgebra Linear Contemporânea. Bookman.

Chen, Z., Zhu, Q., and Soh, Y. C. (2016). Smartphone inertial sensor-based indoor localization and tracking with ibeacon corrections. IEEE Transactions on Industrial Informatics, 12(4):1540-1549.

Chen, Z., Zou, H., Jiang, H., Zhu, Q., Soh, Y. C., and Xie, L. (2015). Fusion of wifi, smartphone sensors and landmarks using the kalman filter for indoor localization. Sensors, 15(1):715-732.

Correa, A., Barcelo, M., Morell, A., and Vicario, J. L. (2017). A review of pedestrian indoor positioning systems for mass market applications. Sensors.

Gomes, R., Alencar, M., Fonseca, I., and Lima Filho, A. (2013). Desafios de redes de sensores sem fio industriais. 4:16-27.

Jin, Y., Toh, H.-S., Soh, W. S., and Wong, W.-C. (2011). A robust dead-reckoning pedestrian tracking system with low cost sensors. pages 222-230.

Junsheng, H. (2017). Wireless industrial indoor localization and its application. Master's thesis, The Artitic University of Norway.

Kent, L. T. (2017). Distância média do passo de corrida. http: / / www • ehow • com . br/distancia-media-passo-corrida-info_8070/.

Khalajmehrabadi, A., Gatsis, N., and Akopian, D. (2017). Modern wlan fingerprinting indoor positioning methods and deployment challenges. IEEE comumunications Surveys \& Tutorials, $19^{\circ}: 1974-2002$.

Koo, B., Lee, S., Kim, S., and Sin, C. (2014). Integrated pdr/fingerprinting indoor location tracking with outdated radio map. pages $1-5$.

Liu, J., Chen, R., Pei, L., Guinness, R., and Kuusniemi, H. (2012). A hybrid smartphone indoor positioning solution for mobile lbs. Sensors, 12(12):17208-17233.

Makki, A., Siddig, A., Saad, M., and Bleakley, C. (2015). Survey of wifi positioning using time-based techniques. Computer Networks, 88:218 - 233.

Milette, G. and Stroud, A. (2012). Professional Android Sensor Programmiing. Wrox.

Ojeda, L. and Borenstein, J. (2007). Personal dead-reckoning system for gps-denied environments. pages $1-6$.

Radu, V. and Marina, M. K. (2013). Himloc: Indoor smartphone localization via activity aware pedestrian dead reckoning with selective crowdsourced wifi fingerprinting. pages 1-10.

Rappaport, T. S. (2001). Wireless Communications: Principles and Practice. Upper Saddle River, NJ, USA, Prentice Hall PTR, 2nd edition. 